\title{
SPECTRAL CHARACTERISTICS OF ANISOTROPIC DIELECTRIC DISK RESONATOR WITH IMPERFECT CONDUCTING END WALLS
}

\author{
Yu.V. Prokopenko, Yu.F. Filippov \\ Usikov Institute of Radiophysics and Electronics, National Academy of Sciences \\ 12, Akad. Proskura St., Kharkov, 61085, Ukraine \\ Tel.: (0572) 448593, E-mail: prokopen@ire.kharkov.ua
}

\begin{abstract}
The electrodynamic analysis of a dielectric disk resonator with imperfect conducting end walls is carried out at excitation of "whispering gallery" oscillations. The possibility of solving the inverse problem is considered: it consists in the determination of permittivity tensor components of anisotropic uniaxial crystal. The spectral characteristics of teflon disk resonator with copper end surfaces are studied in the $8 \mathrm{~mm}$ wave band.
\end{abstract}

\section{INTODUCTION}

Open cylindrical resonators excited by the lowest-type oscillations and made of a material with a high permittivity are widely used for making stable microwave generators and filters. The resonator with impedance end walls permits measurements of low-magnitude thin-film high- $T_{c}$ superconductor surface resistance in a low-frequency band of microwave range.

Now dielectric resonators with low permittivities $(2 \div 10)$ are widely applied. They are excited on higher types of oscillations, which are characterized by high azimuthal indexes. In these resonators the excited oscillations are formed by waves incident on the cylindrical surface at very small angles. This decreases the energy radiation of the resonator. The oscillations are known as "whispering gallery"-type oscillations. A high Q-factor of these quasi-optical resonators determines their application in the devices for millimetric wavelengths. They are also used for study of high- $T_{c}$ superconductor properties.

\section{DIELECTRIC DISK RESONATOR}

In the dielectric disk resonator (DDR) made from a uniaxial single crystal with the anisotropic axis parallel to the resonator longitudinal axis the permittivity tensor has the form

$$
\left[\varepsilon_{i j}\right]=\left\{\begin{array}{ccc}
\delta_{i j}, & & \\
\left(\begin{array}{ccc}
\varepsilon_{\perp} & 0 & 0 \\
0 & \varepsilon_{\perp} & 0 \\
0 & 0 & \varepsilon_{z}
\end{array}\right), r \leq r_{0}
\end{array}\right.
$$

where $\varepsilon_{z}$ and $\mathcal{E}_{\perp}$ are the components of $\left[\mathcal{E}_{i j}\right]$ in parallel and perpendicular directions to the crystal optical axis; $r_{0}$ is the radius of resonator; $\delta_{i j}$ is the Kronecker symbol.

The fields of monochromatic oscillations in such a resonator are described by the expressions [1]:

$$
\begin{array}{lll}
E_{z}=D_{n} G_{E}(r) \cos \left(k_{z} z\right) e^{i(n \varphi-\omega t)} ; & H_{z}=C_{n} G_{H}(r) \sin \left(k_{z} z\right) e^{i(n \varphi-\omega t)} \\
q^{2} E_{\varphi}=\frac{1}{r} \frac{\partial^{2} E_{z}}{\partial \varphi \partial z}-i k \frac{\partial H_{z}}{\partial r} ; & q^{2} E_{r}=\frac{\partial^{2} E_{z}}{\partial r \partial z}+i \frac{k}{r} \frac{\partial H_{z}}{\partial \varphi} ; \\
q^{2} H_{\varphi}=i k \varepsilon_{\perp} \frac{\partial}{\partial r} E_{z}+\frac{1}{r} \frac{\partial^{2} H_{z}}{\partial \varphi \partial z} ; & q^{2} H_{r}=\frac{\partial^{2} H_{z}}{\partial r \partial z}-i \frac{k \varepsilon_{\perp}}{r} \frac{\partial E_{z}}{\partial \varphi} .
\end{array}
$$

Here $G_{j}=J_{n}\left(q_{j} r\right)$ at $r \leq r_{0}$ or $G_{j}=\frac{J_{n}\left(q_{j} r_{0}\right)}{H_{n}^{(1)}\left(q_{0} r_{0}\right)} H_{n}^{(1)}\left(q_{0} r\right)$ at $r \geq r_{0}$, where subscript $j$ takes the value $E$ or $H$; $k=\omega / c$; the azimuth index $n=0 ; 1 ; 2 ; \ldots, k_{z}$ is the axial component of wave vector, $q_{H}=\sqrt{\varepsilon_{\perp} k^{2}-k_{z}^{2}}$, 
$q_{E}=\sqrt{\varepsilon_{z} / \varepsilon_{\perp}} q_{H}$ and $q_{0}=\sqrt{k^{2}-k_{z}^{2}}$ are the radial components of wave vector inside and outside the dielectric, respectively; $C_{n}$ and $D_{n}$ are constants; $q^{2}=q_{H}^{2}$ for $r \leq r_{0}$ and $q^{2}=q_{0}^{2}$ for $r \geq r_{0}$.

The frequency $\omega_{p}=\omega_{p}^{\prime}-i \omega_{p}^{\prime \prime}\left(\omega_{p}^{\prime \prime} \geq 0\right)$ for the $p$-mode resonance oscillation is given by the solution of the characteristic equation:

$$
\left(\alpha_{H}-\alpha_{0}\right)\left(\varepsilon_{z} \alpha_{E}-\alpha_{0}\right)=a^{2}
$$

where $\alpha_{j}=\frac{1}{q_{j} r_{0}} \frac{J_{n}^{\prime}\left(q_{j} r_{0}\right)}{J_{n}\left(q_{j} r_{0}\right)} ; \alpha_{0}=\frac{1}{q_{0} r_{0}} \frac{H_{n}^{(1)^{\prime}}\left(q_{0} r_{0}\right)}{H_{n}^{(1)}\left(q_{0} r_{0}\right)} ; a=\frac{k k_{z} n}{q_{0}^{2} q_{H}^{2} r_{0}^{2}}\left(1-\varepsilon_{\perp}\right)$; the prime denotes the derivative with respect to the argument; $J_{n}(x)$ and $H_{n}^{(1)}(x)$ are the Bessel and first-kind Hankel cylindrical functions of the $n$-th order. The index $p$ characterizes the triple index $n s m$, with $n, s$ and $m$ being the azimuthal, radial and axial indexes, respectively.

For the disk resonator with ideally conducting infinite end surfaces (Fig. 1) the axial component of wave vector is given by the expression $k_{z}=m \pi / l$, where $l$ is the resonator height, $m=0,1,2, \ldots$.

In the DDR there are independent EH and HE-mode oscillations. In the case when the following condition

$$
\left|\left(\alpha_{H}-\alpha_{0}\right)^{1 / 2}\left(\varepsilon_{z} \alpha_{E}-\alpha_{0}\right)^{-1 / 2}\right|>>1
$$

is realized, the eigenoscillations of the resonator will be of the HE type, and the $D_{n}$ constant will be found from the energization condition, and $C_{n}=-D_{n} \frac{J_{n}\left(q_{E} r_{0}\right)}{J_{n}\left(q_{H} r_{0}\right)} \frac{a}{\alpha_{H}-\alpha_{0}}$. Otherwise, the eigenoscillations will be of the EH type, for which the $C_{n}$ constant will be determined from the energization condition, and $D_{n}=-C_{n} \frac{J_{n}\left(q_{H} r_{0}\right)}{J_{n}\left(q_{E} r_{0}\right)} \frac{a}{\varepsilon_{z} \alpha_{E}-\alpha_{0}}$.

The rigorous theory is lacking for the case when the resonator end planes are non-ideally conducting. The frequencies of resonance oscillations in such DDR were calculated by the magnetic-wall method, the variational methods and the method of partial areas with application of an appropriate approximation of electromagnetic field distribution outside the dielectric and with the registration of singularity field behaviour near the dielectric edges (see Tzibisov et al [2] and Lee and Kin [3]). In papers by Filippov and Kharkovsky [4] and Barannik et al [5] it has been suggested to use an experimental identification of resonance oscillations with azimuthal and radial indexes. The radial and axial components of wave vector are thus described by the solutions of the above-mentioned characteristic equation (1), and the relation $l_{\text {eff }}=m \pi / k_{z}$ introduces an effective resonator height.

\section{DDR WITH IMPEDANCE END WALLS}

The square-law relation is obtained from the set of Maxwell's equations, which describe resonance oscillations in the resonators with both ideal and imperfect conducting end walls. Using the condition of a radiated damped wave at $r \rightarrow \infty$, and the continuity of tangential components of electromagnetic field strengths on the resonator curvilinear surface $r=r_{0}$, and also the impedance condition on the end walls, Prokopenko and Filippov [6] came to the integral equation which define the resonance frequency shift as a result of the influence of imperfect conductivity of DDR end walls:

$$
\left(\omega-\omega_{p}^{*}\right) \int_{V}\left(\vec{H} \vec{H}_{p}^{*}+\vec{E}\left[\varepsilon_{i j}\right] \vec{E}_{p}^{*}\right) d V=i \frac{c^{2}}{2 \pi} \zeta \int_{S}\left[\vec{e}_{z}\left[\vec{e}_{z} \times \vec{H}\right] \vec{H}_{p}^{*} d S\right.
$$

Here $\vec{e}_{z}$ is the basis vector of longitudinal resonator axes; $\omega, \vec{E}$ and $\vec{H}$ are, respectively, the frequency and the vectors of electrical and magnetic field strengths of the resonator with the final conducting end walls; $\omega_{p}, \vec{E}_{p}$, 
$\vec{H}_{p}$ are the resonance frequency and the fields of $p$-mode oscillation in the DDR with ideally conducting end walls (* designates the complex conjugation). On the left, the integration is over whole volume $V$, and on the right, it is carried out on the end plane surfaces $S$ characterized by the surface impedance $\zeta=R_{S}+i X_{S}$.

Using the representation of vectors $\vec{E}$ and $\vec{H}$ as a linear combination of corresponding DDR eigenfields $\vec{E}_{p}$ and $\vec{H}_{p}$ with ideally conducting end walls, we obtain the following set of equations:

$$
\left(\omega-\omega_{p}^{*}\right) W_{p} \beta_{p}=-i \zeta \sum_{p^{\prime}} I_{p_{p}}^{2} \beta_{p^{\prime}}
$$

Here $\beta_{p^{\prime}}$ is the expansion constant; $W_{p}=\frac{1}{8 \pi} \int_{V}\left(\vec{H}_{p} \vec{H}_{p}^{*}+\vec{E}_{p}\left[\varepsilon_{i j}\right] \vec{E}_{p}^{*}\right) d V$ is the electromagnetic field energy produced by the $p$-mode oscillation in the resonator with ideally conducting end walls. The parameter $I_{p^{\prime} p}^{2} \zeta=\frac{c^{2}}{16 \pi^{2}} \zeta \int_{S}\left[\vec{e}_{z}\left[\vec{e}_{z} \times \vec{H}_{p^{\prime}}\right] \vec{H}_{p}^{*} d S\right.$ takes into account the losses in the end walls of final conductivity and describes the interaction between the modes in the DDR. The compatibility condition of the homogeneous linear equations system relative to $\beta_{p^{\prime}}$ gives the characteristic equation for the calculation of eigenfrequencies in the DDR with imperfect conducting end walls:

$$
\operatorname{det}\left\|\left(\omega-\omega_{p}^{*}\right) \delta_{p^{\prime} p}+i \zeta \frac{I_{p^{\prime} p}^{2}}{W_{p}}\right\|=0
$$

The given relation determines the resonance frequency shift of DDR with imperfect conducting end walls relative to the eigenfrequency of the resonator with ideally conducting end surfaces for the respective oscillation type. The change in the resonance-frequency real part is determined by the imaginary part of surface impedance (reactance) $X_{S}$. The nondissipative energy accumulated in the surface strata of DDR end walls depends on the reactance magnitude. The surface resistance $R_{S}$ determines the average Joule heat losses per cycle $I_{p p}^{2} R_{S}$ in the end impedance surfaces of resonator.

For a small surface impedance $\zeta(\zeta<<1)$ and with neglection of interaction between the modes $\left(I_{p^{\prime} p}^{2}=0\right.$ at $p^{\prime} \neq p$ ) relation (2) takes the form

$$
\left(\omega-\omega_{p}^{*}\right)+i \zeta \frac{I_{p p}^{2}}{W_{p}}=0
$$

where $I_{p p}$ is the surface current in the resonator end planes with final conductivity. This current is generated by penetration of electromagnetic wave in the planes. The obtained relation makes it possible to determine the surface impedance $\zeta$ of the resonator end walls at the DDR resonance frequency $\omega$ measured in experiment.

\section{SPECTRAL CHARACTERISTICS OF DDR}

The spectral characteristics of the DDR with impedance end surfaces are shown in Fig. 2 at $R_{S}=4 \times 10^{-2}$ Ohm (for copper). In calculations and experimental studies we used the DDR made from a teflon sheet ( $\varepsilon_{\perp}=\varepsilon_{z}=2.04$ ) and being $3.9 \mathrm{~cm}$ in the radius and $0.71 \mathrm{~cm}$ in the height. The frequency dependences of the slow-down factor $\xi=n / k^{\prime} r_{0}-1$ have been obtained from relation (3) for the reactance $X_{S}=R_{S}$. The resonator quality factor $Q_{0}$ values was determined by Barannik's et al expression [5]. Fig. 2 shows the results for the DDR with EH and HE-mode oscillations which have the radial index $s=1 ; 2$ and the axial index $m=0 ; 1$ within the azimuthal indexes $n=30 \div 39$. 
The resonance frequency deviation of the resonator with copper end walls from respective eigenfrequency values of the DDR with ideally conducting surfaces was $(10 \div 15) \mathrm{MHz}$. The resonance-oscillations power losses in copper end surfaces have led to a decrease of resonator basic Q-factor by factors of 10 to 100 .

\section{CONCLUSION}

The integral equation that describes the influence of end-wall final conductivity on resonance oscillations is obtained and investigated. The slow-down and Q-factor dependences of teflon DDR with copper end walls are given. The solution of the self-consistent set of equations on the basis of the characteristic relation (1) and experimentally measured azimuthal and axial indexes at resonance frequencies of "whispering gallery" oscillations determine the permittivity tensor components $\left[\varepsilon_{i j}\right]$ of the uniaxial crystal that has its optical axis coinciding with the direction of the DDR longitudinal axis. It is necessary to note that the solution of the integral equation (3) allows determining the frequency dependence of the surface impedance at known experimental values of resonance frequencies. The approach developed by us can be used for studying microwave characteristics of conducting materials, including superconducting films.

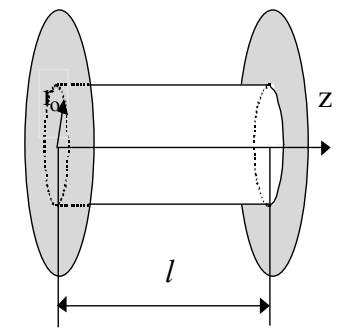

Figure 1. DDR with conducting end walls

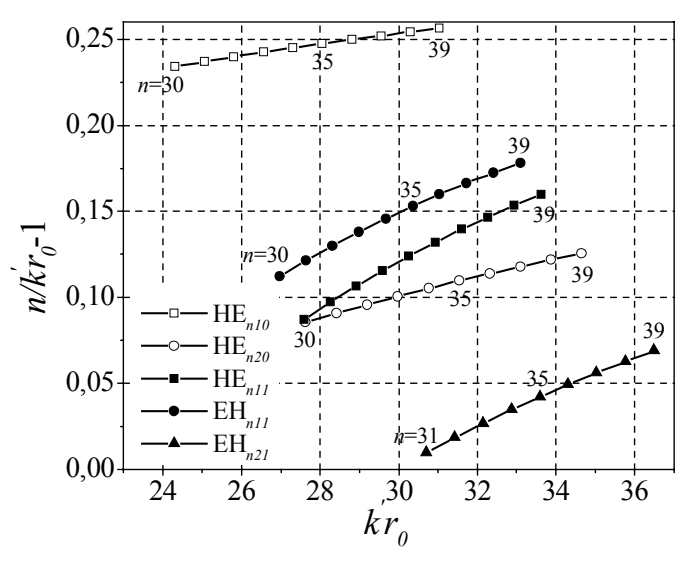

a)

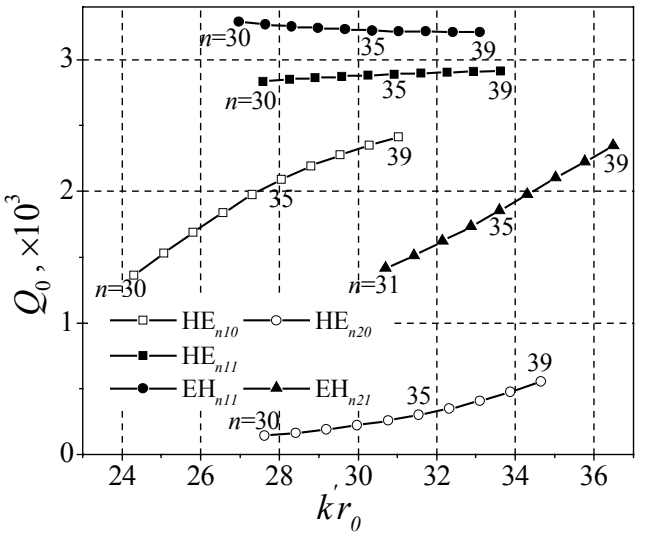

b)

Figure 2. Slow-down (a) and Q-factor (b) curves for the DDR with copper end walls

\section{REFERENCES}

1. Egorov V.N., Malzseva I.N. Oscillations in anisotropic dielectric resonator with end reflectors. 1984. Elektronnaya tehnika. Seriya I. Elektronika SVCh. No. 1. P. 3-8. (in Russian).

2. Tzibisov K.N., Borisov S.A., Besborodov Yu.M. Calculation methods of resonance frequencies in dielectric resonators. 1981. Zarubezshnaya radioelektronika. No. 1. P. 21-33. (in Russian).

3. Lee J., Kin Y.S. A new method of accurately determining resonant frequencies of cylindrical and ring dielectric resonators. 1999. IEEE Trans. on MTT. Vol. 47, No. 6. P.706-708.

4. Filippov Yu.F., Kharkovsky S.N. Spectra of resonance oscillations in disk dielectric resonator. 1990. Izvestiya Vuzov. Radiofizika. Vol. 33, No. 11. P. 1304-1308. (in Russian).

5. Barannik A.A., Prokopenko Yu.V., Filippov Yu.F., Cherpak N.T. Quasi-optical dielectric resonator with uniaxial anisotropy. Measurement of dielectric and conductors characteristics. 2000. Radiofizika i Elektronika. - Kharkov: Institute of Radiophysics and Electronics NAS of Ukraine. Vol. 5, No. 3. P. 104-109. (in Russian).

6. Prokopenko Yu.V., Filippov Yu.F. Influence of final conductivity of end walls on spectral and power characteristics of anisotropic dielectric disk resonator. 2001. Radiofizika i Elektronika. - Kharkov: Institute of Radiophysics and Electronics NAS of Ukraine. Vol. 6, No. 1. P. 49-55. (in Russian). 